\title{
Antibacterial and cytotoxic effect of honey mediated copper nanoparticles synthesized using ultrasonic assistance
}

\author{
Nur Afini Ismail ${ }^{\mathrm{a}}$, Kamyar Shameli ${ }^{\mathrm{a}, *}$, Magdelyn Mei-Theng Wong ${ }^{\mathrm{b}}$, Sin-Yeang Teow ${ }^{\mathrm{b}}$, \\ Jactty Chew ${ }^{c}$, Siti Nur Amalina Mohamad Sukri ${ }^{\mathrm{a}}$ \\ ${ }^{a}$ Department of Environment and Green Technology, Malaysia-Japan International Institute of Technology, Universiti Teknologi Malaysia, Jalan Sultan Yahya Petra, \\ 54100 Kuala Lumpur, Malaysia \\ ${ }^{b}$ Department of Medical Sciences, School of Healthcare and Medical Sciences, Sunway University, Jalan Bandar Sunway, 47500, Selangor Darul Ehsan, Malaysia \\ ${ }^{\mathrm{c}}$ Department of Biological Sciences, School of Science and Technology, Sunway University, Jalan Bandar Sunway, 47500, Selangor Darul Ehsan, Malaysia
}

\section{A R T I C L E I N F O}

\section{Keywords:}

Antibacterial

Cytotoxicity assay

Copper nanoparticle

Honey mediated

Sonochemical

\begin{abstract}
A B S T R A C T
In this study, a comparative study of effect using honey on copper nanoparticles (Cu-NPs) via simple, environmentally friendly process and inexpensive route was reported. Honey and ascorbic acid act as stabilizing and reducing agents with the assistance of sonochemical method. The products were characterized using UV-visible (UV-vis) spectroscopy, X-Ray Diffraction (XRD), High-Resolution Transmission Electron Microscopy (HRTEM), Field-Emission Scanning Electron Microscopy (FESEM) and Fourier Transform Infrared (FTIR) spectroscopy. The reddish brown colour demonstrated the formation of Cu-NPs and UV-visible proved the plasmon resonance of Cu-NPs. XRD also confirmed a highly pure Cu-NPs obtained with absence of copper oxide in which the structure is crystalline. The spherical size of the Cu-NPs was acquire in the presence of honey which is $3.68 \pm 0.78 \mathrm{~nm}$ with narrow particle distribution. The antibacterial activity was seen against gram-positive and gram-negative bacteria which are Enterococcus faecalis (E. faecalis) and Escherichia coli (E. coli). At higher concentration of Cu-NPs, they were more effective in killing both bacteria. The Cu-NPs without and with honey exhibited toxicities toward normal and cancerous cells. However, Cu-NPs without honey showed more potent killing activity against normal and cancer cells.
\end{abstract}

\section{Introduction}

Nanotechnology emerges as one of the alternative technologies in addressing problems that arise nowadays particularly pertaining to environmental issues [1]. It also has various medical and biomedical applications such as the production of nanoparticles and carbon nanotubes for bio-imaging, cancer therapy, drug delivery and antibacterial [2-4]. Nanoparticle production, particularly metallic and metal oxide nanoparticles, is attracting vast interest from scientists in recent years due to the unique characteristics such as having surface plasmon resonance (SPR) and optical properties [5]. They are also actively used for biosensor, electrical, catalytic and antimicrobial applications [6-9]. The size of nanomaterials typically ranges from 1 to $100 \mathrm{~nm}$. Generally, nanoparticles could be classified as fine particles which has the size ranging from 100 to $2500 \mathrm{~nm}$ while ultrafine particles possess the size from 1 to $100 \mathrm{~nm}$ [10].

Gold-, platinum-, silver-, and copper-nanoparticles (Cu-NPs) are a few relatively well studied nanoparticles. Among them, Cu-NPs have special characteristics such as low cost, less toxic, high surface area to volume ratio, heat transfer properties and high friction of atoms $[11,12]$. These characteristics are mainly resulted by their physical properties such as size, shape, composition, morphology and crystalline phase [13]. Cu-NPs also have abundant sources, easy preparation and flexibility in modifying into various shape of nano-sized dimension [14].

Cu-NPs can be implemented in several applications such as biomedical, optical, biosensor, catalytic and energy application [15-19]. In an important biomedical application which is drug delivery system and molecular doping, Cu-NPs play particularly important role as vehicles to deliver the compounds to the target sites [20]. Moreover, copper also is a multifunctional antibacterial agents [21]. Cu-NPs have been demonstrated to show potent inhibition toward various bacterial species [22-25]. Various studies showed that Cu-NPs could bind and conjugate with biomolecules like proteins, enzymes and DNA [20,26], indicating the potentials of Cu-NPs to be used as 'nano-drugs' [27].

There are various ways to synthesize Cu-NPs using physical and

\footnotetext{
* Corresponding author.

E-mail address: kamyarshameli@gmail.com (K. Shameli).
} 